\section{COMMENTS AND REPLIES}

\section{Online: GSA Today, Comments and Replies Published Online: November 2017}

\section{Reply}

\section{Reply to the comment by Dr. William Turner}

Terry L. Pavlis and Kelsey A. Mason, Dept. of Geological Sciences, The University of Texas at El Paso, El Paso, Texas 79968, USA

We enthusiastically welcome the chance to reply to this comment because the attitude about digital mapping in the field expressed by Dr. Turner is a common one. There have been several heated debates on this issue in online forums and we, along with other colleagues, have had numerous discussions on the issues raised here. There is no question that many geologists make fabulous maps using traditional methods. The real issue is that better methods are now available. Indeed, we wonder how many more people would have become skilled mappers had modern tools been available to them as students. These issues were a major driver that led us to write this paper.

That said, we would make this suggestion: Anyone who hasn't actually tried any of these modern techniques has no idea how dramatically they can improve both field efficiency and accuracy of geologic mapping. Note that this statement is NOT made from ignorance of what is involved in field geology - the lead author of this paper has more than 40 years of field experience, having worked at a range of scales in many tectonic settings using techniques ranging from blank paper pace-compass mapping to the modern 3D mapping described in this paper. He has also taught dozens of field geology classes using both paper and digital mapping techniques. From that experience, we can unequivocally state that even basic 2D digital mapping dramatically improves field efficiency by providing: (a) georeferenced map bases that can be directly overlain (no paper shuffling needed); (b) GPS positioning (no need to waste time determining your exact location); (c) automated plotting of symbols (no protractor needed); and (d) graphical interfaces that speed field data collection/display (no need to spent time sharpening pencils, accidently erasing a line, etc.). Adding GIS functionality further contributes to making a map more than a static object when map objects carry attributes that contain critical information; e.g. when drawing a line, a field worker might add comments ranging from "I am guessing where this line is" to "I walked every inch of this line marking it with a GPS." Moreover, accuracy in general is something that now can be quantified. Dr. Turner may have mapped at 1:5000 and states he could easily place a contact to $\pm 2 \mathrm{~m}$, but our response to that is: Can you prove that quantitatively? Our experience is the answer is "no" for traditional methods. Now, however, you can answer that question quantitatively because any other geologist could retrace your steps exactly with GPS and test that hypothesis of reproducibility. All of these factors turn modern geologic maps into data rather than traditional maps where the distinctions between observation and interpretation are uncertain.

These are just a few of the benefits of basic digital mapping, but as we indicated in our paper (GSA Today, v. 27, no. 9, https://doi. org/10.1130/GSATG313A.1), basic digital mapping pales in comparison to what can be done with true 3D mapping. One important point, however, is that Dr. Turner actually misses a key point of our paper about our view of the future: We in no way were promoting "armchair geology" and fully recognize that boots-onthe-ground work (like his fossil example) remains the critical essence of our science. Remote sensing techniques simply free up field time for what is most important: establishing basic geologic field relationships that build an understanding of geologic history and geologic processes. Improving geometric accuracy eliminates many classic ambiguities of field geology and allows more time on the ground for assessing real geologic problems rather than the question: Is that line really in the right place? Finally, we reiterate that full 3D techniques are not always needed. If it is as flat as Kansas and outcrop is poor, 3D techniques are a waste of time. In steep terrain, however, this technology is transformative.

The bottom line is, to rephrase a conclusion in the paper, we are at the dawn of a new age of field geology that will allow us to do things that were impossible only a few years ago. For those who haven't tried digital techniques, give it a try. It might be frustrating initially, but as you learn the software you'll be amazed what you can do.

Manuscript ReCeived 19 Oct. 2017

MANUSCRIPT ACCEPTED 24 OCT. 2017 